\title{
NutriDia Brasil: retrato dos cuidados nutricionais em um hospital de alta complexidade do Rio Grande do Sul
}

\author{
NutriDia Brasil: nutritional care snapshot in a high complexity hospital of Rio Grande do Sul
}

\section{DOI: $10.37111 /$ braspeni.2020353004}

\section{Marilene Fleck de Oliveira'}

Maria Cristina Zanchim²

Carolina Benvenuti de Mattos ${ }^{3}$

Vanuza Burille'

Tatiana Pacheco Rodrigues ${ }^{4}$

Silvana Paiva Orlandi ${ }^{5}$

Maria Cristina Gonzalez

\section{Unitermos:}

Avaliação nutricional. Desnutrição. Ingestão de alimentos.

\section{Keywords:}

Nutrition assessment. Malnutrition. Eating.

\section{Endereço para correspondência:}

Maria Cristina Zanchim

Rua 7 de agosto, 510, apto. 504 - Centro - Passo

Fundo, RS, Brasil - CEP 99025-030

E-mail: cris_zanchim@upf.br

\section{Submissão}

6 de janeiro de 2020

\section{Aceito para publicação}

1 de setembro de 2020

\begin{abstract}
RESUMO
Introduçãa: A desnutrição hospitalar é considerada um problema de saúde pública, cuja prevalência varia de $40 \%$ a $60 \%$ na admissão, alcançando cifras de $80 \%$ durante o curso da internação. Neste contexto, o projeto nutriDia Brasil objetiva evidenciar problemas nutricionais que os pacientes hospitalizados possuem, assim como recursos disponíveis para o manejo e o conhecimento dos profissionais da saúde a esse respeito. Método: Estudo longitudinal observacional, com a utilização de dados da equipe multiprofissional de terapia nutricional do Hospital São Vicente de Paulo, de Passo Fundo, RS, instituição participante do nutriDia Brasil, em novembro de 2017. Para composição do estudo avaliaram-se variáveis como sexo, idade, diagnóstico, tempo de hospitalização, índice de massa corporal (IMC), perda de peso, identificação do risco nutricional, tipo de terapia nutricional, aceitação de dieta, consumo de alimentos fora da rotina hospitalar e desfecho clínico após 30 dias. Resultados: Foram avaliados 136 pacientes, com idade média de $57 \pm 3,2$ anos, a maioria do gênero masculino (53\%). As principais causas da hospitalização foram as doenças do aparelho osteomuscular $(21,1 \%)$, circulatórias $(15,8 \%)$, digestivas $(13,9 \%)$ e geniturinárias (10\%). Com relação ao estado nutricional, a média do IMC foi de $25,9 \pm 4,8 \mathrm{~kg} / \mathrm{m}^{2}$ e a perda de peso esteve referenciada em $55 \%$ dos casos, com redução média de $6,7 \pm 2,1 \mathrm{~kg}$. Apresentaram apetite reduzido $39,2 \%$ dos avaliados e apenas $51,9 \%$ conseguiram ingerir toda a dieta hospitalar prescrita. Dentre as causas da redução da ingestão alimentar, $13,2 \%$ referiram sintomas como náuseas ou vômitos e $9,1 \%$, perda de apetite. Recebiam dieta oral exclusiva $71,3 \% ; 17,4 \%$ faziam uso de suplementos nutricionais associados à alimentação hospitalar e 11,3\%, nutrição artificial. Conclusão: A perda ponderal, a inadequação da ingestão alimentar e a presença de desvios nutricionais foram os fatores evidenciados mais relevantes, sugerindo a necessidade de implementação de melhorias que contribuam para uma assistência segura e de qualidade ao paciente.
\end{abstract}

\section{ABSTRACT}

Introduction: Hospital malnutrition is considered a public health problem, whose prevalence ranges from $40 \%$ to $60 \%$ on admission, reaching figures of $80 \%$ during the course of hospitalization. In this context, the nutriDia Brasil project aims to highlight nutritional problems that hospitalized patients have, as well as resources available for the management and knowledge of health professionals in this regard. Methods: Longitudinal observational study, using data from the multiprofessional nutritional therapy team at Hospital São Vicente de Paulo, in Passo Fundo, RS, an institution participating in nutriDia Brasil, in November 2017. For the composition of the study, variables such as gender, age, diagnosis, length of hospital stay, body mass index (BMI), weight loss, identification of nutritional risk, type of nutritional therapy, acceptance of diet, consumption of food outside the hospital routine and clinical outcome after 30 days. Results: 136 patients were evaluated, with a mean age of 57 \pm 3.2 years, most of whom were male $(53 \%)$. The main causes of hospitalization were diseases of the musculoskeletal system (21.1\%), circulatory (15.8\%), digestive $(13.9 \%)$ and genitourinary $(10 \%)$. Regarding nutritional status, the mean BMI was $25.9 \pm 4.8 \mathrm{~kg} / \mathrm{m}^{2}$ and weight loss was reported in $55 \%$ of cases, with an average reduction of $6.7 \pm 2.1 \mathrm{~kg}$. $39.2 \%$ of those evaluated and only $51.9 \%$ managed to ingest the entire hospital diet prescribed. Among the causes of reduced food intake, $13.2 \%$ reported symptoms such as nausea or vomiting and $9.1 \%$ loss of appetite. $71.3 \%$ received an exclusive oral diet; $17.4 \%$ used nutritional supplements in combination with hospital foods and $11.3 \%$ received artificial nutrition. Conclusion: Weight loss, inadequate food intake and the presence of nutritional deviations were the most relevant factors, suggesting the need to implement improvements that contribute to safe and quality care for the patient.

2. Mestre em Envelhecimento Humano pela Universidade de Passo Fundo (UPF); Especialista em Terapia Nutricional pela SBNPE/BRASPEN; Docente do Curso de Nutrição da UPF, Passo Fundo, RS, Brasil.

3. Docente do Curso de Nutrição da Universidade de Passo Fundo (UPF), Mestre em Endocrinologia, Metabologia e Nutrição pela Universidade Federal do Rio Grande do Sul (UFRGS), Passo Fundo, RS, Brasil.

4. Pós-graduada em Terapia Nutricional Parenteral e Enteral pela Pontifícia Universidade Católica do Rio Grande do Sul (PUCRS); Pós-graduanda em Formação Pedagógica para a Educação Profissional. Nutricionista membro da Equipe Multiprofissional de Terapia Nutricional do Hospital São Vicente de Paulo (HSVP), Passo Fundo, RS, Brasil

5. Departamento de Nutrição. Universidade Federal de Pelotas, Doutora em Epidemiologia pela Universidade Federal de Pelotas, Pelotas, RS, Brasil.

6. Programa de Pós-Graduação em Saúde e Comportamento, Universidade Católica de Pelotas e Programa de Pós-Graduação em Nutrição e Alimentos, Universidade Federal de Pelotas; Doutora em Epidemiologia pela Universidade Federal de Pelotas, Pelotas, RS, Brasil. 


\section{INTRODUÇÃO}

A desnutrição hospitalar manifesta-se mundialmente, sendo considerada um grande problema de saúde pública. Sua prevalência estimada em adultos varia de $40 \%$ a $60 \%$ no momento da admissão, alcançando cifras de até $80 \%$ durante o curso da internação'. No Brasil, o estudo multicêntrico denominado IBRANUTRI (Inquérito Brasileiro de Avaliação Nutricional) avaliou 4000 mil pacientes internados em hospitais da rede pública, revelando que aproximadamente $48 \%$ apresentavam algum grau de desnutrição, sendo $12 \%$ destes gravemente desnutridos ${ }^{2}$.

Nos últimos anos, muitas publicações científicas apontam a desnutrição como a principal responsável pelos altos índices de morbidade devido a cicatrização lenta de feridas, altas taxas de infecção, maior tempo de permanência hospitalar, principalmente em unidades de tratamento intensivo, custos com a assistência médica e mortalidade. Desta forma, pelos resultados desfavoráveis a ela associados, tem crescido o interesse pela investigação do estado nutricional dos pacientes internados ${ }^{3,4}$.

A desnutrição hospitalar pode ter origem multifatorial, estando associada a situações clínicas e/ou tratamento instituído. Porém, diversos fatores contribuem para seu agravamento, como a idade avançada, saúde oral prejudicada, isolamento social e de forma contundente, a execução de forma incorreta de condutas básicas na alimentação. A suspensão da alimentação para realização de exames clínicos e procedimentos operatórios, as mudanças na composição, sabor e horário da dieta oferecida no ambiente hospitalar e o desconhecimento por parte da equipe de saúde do impacto negativo da ingestão alimentar deficiente acabam colocando o paciente em risco nutricional ou agravando sua desnutrição prévia ${ }^{5}$.

Segundo Bottoni et al. ${ }^{6}$, a identificação precoce de possíveis alterações nutricionais e a implementação da terapia nutricional tornam-se bastante benéficas, por prevenir as consequências negativas que a privação de nutrientes provoca no prognóstico do paciente. Sabe-se, porém, que existem diversos problemas e limitações na prática clínica, pois cada hospital tem sua rotina estabelecida, tanto em relação ao paciente como ao gerenciamento. Nesses setores, por vezes, não há padronização, o que pode interferir negativamente no planejamento e na atenção dietética prestada ao paciente ${ }^{7}$.

Neste sentido, considerando a necessidade de se conhecer melhor como os profissionais e os serviços de saúde agem em relação aos cuidados nutricionais de seus pacientes, iniciou-se em 2006, na Áustria, um projeto multicêntrico denominado NutritionDay, que se trata de uma auditoria realizada em único dia, com o objetivo de avaliar uma ou várias unidades de um mesmo hospital, tentando evidenciar os problemas nutricionais que seus pacientes possuem, os recursos disponíveis para lidar com os problemas nutricionais, assim como o conhecimento que os profissionais da saúde possuem a esse respeito ${ }^{8}$.

No Brasil, esse projeto foi implantado pela Sociedade Brasileira de Nutrição Parenteral e Enteral (SBNPE) a partir do ano de 2009 e recebeu o nome de NutriDia Brasil. Dessa forma, o objetivo deste estudo foi descrever o retrato dos cuidados nutricionais em pacientes de um hospital geral de alta complexidade do Rio Grande do Sul (RS).

\section{MÉTODO}

Estudo de caráter longitudinal observacional, com a utilização de dados da equipe multiprofissional de terapia nutricional (EMTN) do Hospital São Vicente de Paulo (HSVP), de Passo Fundo, RS, instituição participante do projeto nutriDia Brasil, realizado em novembro de 2017. O hospital necessitou atender a critérios para inclusão na pesquisa, sendo eles, obter um profissional ligado à SBNPE para coordenar o estudo localmente e dispor de recursos humanos próprios para aplicação dos questionários.

No dia da avaliação, coletaram-se os dados em seis enfermarias de internação, sendo avaliados pacientes adultos e idosos ( $\geq 18$ anos), que concordaram em participar do estudo e o fizeram mediante assinatura do Termo de Consentimento Livre e Esclarecido (TCLE), fornecido pela comissão nacional do estudo. Na impossibilidade de assinatura pelo paciente, esta foi realizada pelo familiar responsável da internação.

Para coleta dos dados foram utilizados quatro questionários padronizados, disponíveis no link: http://www. nutritionday.org/index.php?id=346, preenchidos pela equipe do hospital previamente treinada. Os dois questionários iniciais eram destinados à equipe de saúde, com questões sobre a estrutura do hospital/enfermaria, seus recursos humanos, além de informações dos pacientes: estado nutricional, medicações usadas, diagnóstico, presença de comorbidades, uso de terapia nutricional e tempo de internação; e os outros dois foram aplicados à beira leito, diretamente com os pacientes, e continham informações sobre o monitoramento do peso, mobilidade, consumo alimentar e apetite no dia da avaliação. Ainda, após o período de 30 dias, o desfecho clínico dos pacientes foi reavaliado.

As variáveis utilizadas para a composição do estudo em questão foram: sexo, idade, diagnóstico, tempo de hospitalização, peso e altura autorrelatados, índice de massa corporal (IMC), perda de peso nos últimos 3 meses, identificação do risco nutricional, tipo de terapia nutricional, aceitação de dieta, consumo de alimentos fora da rotina hospitalar e desfecho clínico, que é classificado como alta domiciliar, óbito e outros (transferência para outro hospital, reabilitação ou sem definição). 
Os questionários foram computados no site do projeto pela EMTN do HSVP e analisados no programa estatístico Stata (versão 12.0) pela coordenação nacional do NutritionDay no Brasil. Dos relatórios obtidos, foi utilizada uma parte dos dados para a realização deste estudo, sendo as variáveis estudadas descritas por meio de frequências relativas e absolutas. O estudo foi aprovado pelo Comitê de Ética em Pesquisa da Universidade Católica de Pelotas, sob o número do parecer 1.136.58, e pela Comissão de Pesquisa e Pós-Graduação do HSVP.

\section{RESULTADOS}

\section{Dados Demográficos e Clínicos}

Foram avaliados 136 pacientes, com idade média de $57 \pm 3,2$ anos, variando entre 32 a 76 anos, sendo a maioria do gênero masculino (53\%). As principais causas que motivaram a hospitalização foram as doenças do aparelho osteomuscular $(21,1 \%)$, circulatórias $(15,8 \%)$, digestivas (13,9\%) e geniturinárias (10\%); e 87\% tinham como convênio o Sistema Único de Saúde (SUS). A presença de comorbidades foi relatada por $61 \%$ dos avaliados, sendo a insuficiência cardíaca, diabetes e doença pulmonar obstrutiva crônica as mais comuns $(23,6 \%, 13,3 \%$ e $11,2 \%$, respectivamente). Em termos de capacidade funcional, 15,6\% dos pacientes necessitavam de assistência para deambular. A mediana do tempo de internação foi de 17,5 $\pm 4,7$ dias, tendo como desfecho principal a alta hospitalar $(88,2 \%)$. Após monitoramento de 30 dias, $4 \%$ dos pacientes foram readmitidos na mesma instituição (Tabela 1).

\section{Estado Nutricional}

Quanto aos dados antropométricos, o peso, estatura e IMC médios foram de 71,72 $\pm 15,02 \mathrm{~kg}, 166 \pm 9,83 \mathrm{~cm}$ e $25,92 \pm 4,8 \mathrm{~kg} / \mathrm{m}^{2}$, respectivamente. Dentre os pacientes avaliados, 50,6\% relataram terem sido pesados na admissão e quando questionados sobre o estado nutricional pregresso à internação, 54,9\% informaram perda de peso não intencional nos últimos três meses, com uma média de $6,7 \pm 2,1 \mathrm{~kg}$. A prevalência geral de risco de desnutrição ou desnutrição já instalada estava documentada em $34,6 \%$ dos prontuários avaliados (Tabela 1).

\section{Ingestão de Alimentos e Suporte Nutricional}

Dentre os pacientes que participam do nutriDia, uma alta porcentagem recebia dieta oral exclusiva, sendo $41,7 \%$ prescrições de dietas regulares (modificadas na consistência) e $47 \%$ especializadas. Sobre a alimentação domiciliar, a maioria $(60,8 \%)$ relatou consumo normal/ mais que o normal nos sete dias que antecederam a hospitalização, porém após a hospitalização, a redução do
Tabela 1 - Caracterização demográfica, clínica e do estado nutricional dos pacientes de um hospital de alta complexidade, Passo Fundo - RS, 2017. $(n=136)$

\begin{tabular}{lcc}
\hline Variáveis & $\mathbf{n}$ & $\%$ \\
\hline Gênero & 72 & 53,0 \\
Masculino & 64 & 47,0 \\
Feminino & & \\
\hline Diagnóstico clínico & 30 & 22,1 \\
Doenças do aparelho osteomuscular & 22 & 15,8 \\
Doenças do aparelho circulatório & 19 & 13,9 \\
Doenças do aparelho digestivo & 14 & 10,0 \\
Doenças do aparelho geniturinário & 51 & 38,2 \\
Outras & & \\
\hline Presença de comorbidades & 83 & 61,0 \\
Sim & 53 & 39,0 \\
Não & & \\
\hline Desfecho & 120 & 88,2 \\
Alta & 3 & 2,2 \\
Óbito & 13 & 9,6 \\
Outros & & \\
\hline Perda de peso nos últimos 3 meses & 75 & 54,9 \\
Sim & 61 & 45,1 \\
Não & & \\
\hline Inadequação nutricional no prontuário & & \\
Risco nutricional & & \\
Desnutrição & & \\
Não & & \\
\hline & & \\
\hline
\end{tabular}

apetite foi referenciada por $59,7 \%$ dos pacientes. No dia da pesquisa, apenas $51,9 \%$ consumiram toda a refeição principal (desjejum, almoço e/ou jantar), sendo que $26,4 \%$ ingeriram metade da refeição, e $21,7 \%$ comeram cerca de um quarto da refeição ou nada (Tabela 2) e entre os motivos citados para a baixa ingestão ou ingestão nula estavam a presença de náuseas/vômitos, problemas com mastigação/deglutição, a insatisfação com cheiro, sabor ou temperatura da preparação ou a necessidade de jejum para exames ou cirurgias. A consulta de um especialista em nutrição estava detalhada em $68,1 \%$ dos prontuários e, 25,9\% tiveram um plano de intervenção nutricional desenvolvido.

A satisfação geral com a comida oferecida pelo hospital encontra-se demonstrada na Figura 1. Em termos de terapia nutricional, 17,4\% faziam uso de suplementos de proteína/energia em combinação com os alimentos hospitalares e 11,3\% recebiam nutrição artificial (por exemplo, nutrição enteral, parenteral ou combinada enteral e parenteral). 


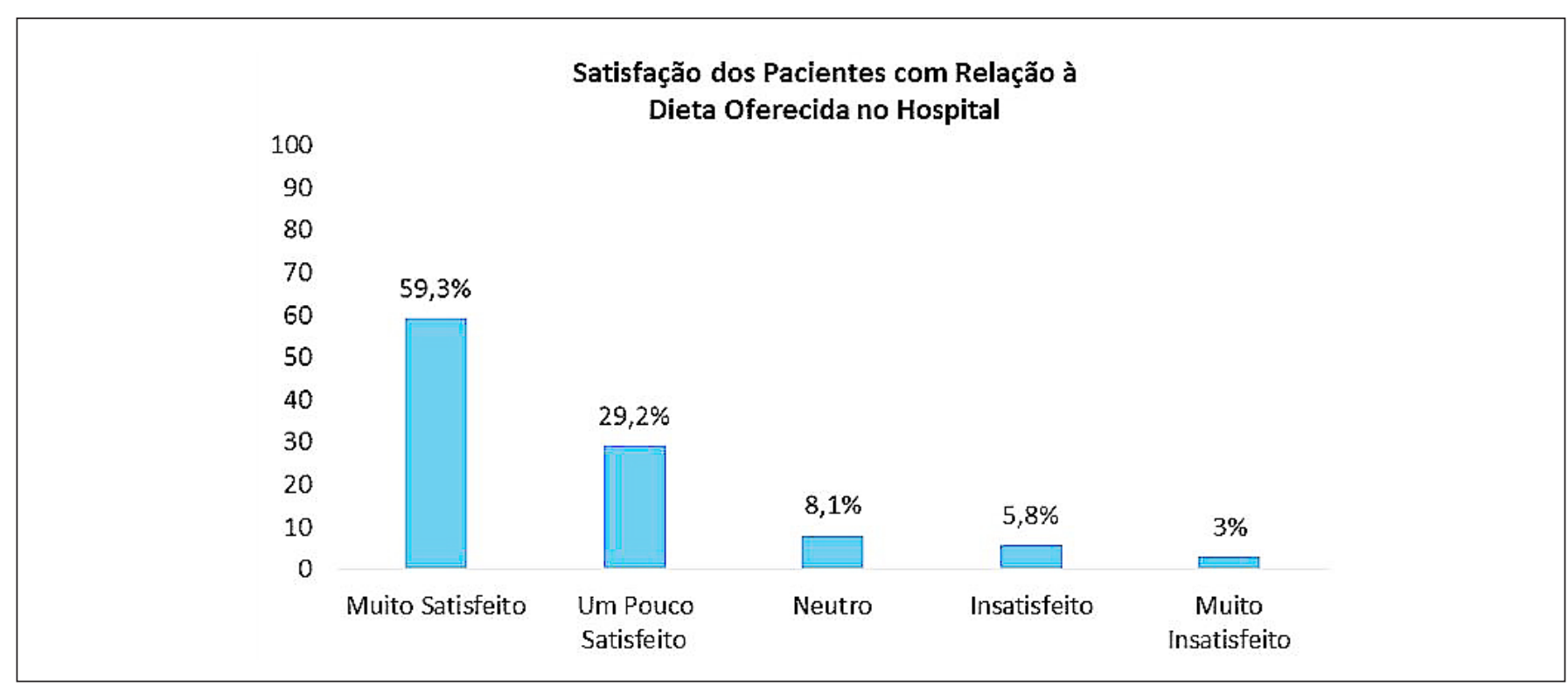

Figura 1 - Percentual da satisfação com a dieta do hospital.

Tabela 2 - Caracterização da ingestão de alimentos e suporte nutricional dos pacientes de um hospital de alta complexidade, Passo Fundo - RS, 2017. $(n=136)$

\begin{tabular}{lcc}
\hline Variáveis & $\mathbf{n}$ & $\%$ \\
\hline Via da alimentação & 121 & 88,7 \\
Oral exclusiva & 9 & 6,6 \\
Enteral exclusiva & 6 & 4,7 \\
Outras & & \\
\hline Alimentação na última semana & 83 & 60,8 \\
Mais que o normal/normal & 18 & 13,1 \\
Quase 3/4 do normal & 19 & 14,2 \\
Quase metade do normal & 16 & 11,9 \\
Menos de 1/4 ou quase nada & & \\
\hline Apetite mudou durante a internação & 81 & 59,7 \\
Sim & 55 & 40,3 \\
Não & & \\
\hline Alimentação na refeição principal & 72 & 51,9 \\
Quase tudo & 36 & 26,4 \\
Metade (1/2) & 17 & 13,9 \\
Um quarto (1/4) & 11 & 7,9 \\
Nada &
\end{tabular}

\section{DISCUSSÃO}

Na população avaliada, houve prevalência de indivíduos do gênero masculino e de adultos, comprovando o encontrado por alguns estudos que avaliaram o estado nutricional de pacientes hospitalizados, como o nutriDia Brasi ${ }^{9}$ realizado na cidade de Pelotas (RS), onde 64,9\% dos avaliados eram homens e por Zheng et al. ${ }^{3}$, na China, no qual $59,3 \%$ eram homens e a idade média de 53,53 $\pm 15,82$ anos. Estes dados podem ser justificados com achados da literatura, que constatam que homens adultos geralmente buscam com menor frequência os serviços de saúde de atenção básica, ficando mais susceptíveis ao desenvolvimento de enfermidades, sobretudo, graves e crônicas, que demandam por maior necessidade de hospitalização ${ }^{10,11}$.

Com relação à presença de comorbidades, 23,6\% dos pacientes foram identificados com insuficiência cardíaca (IC), similar ao encontrado por Saver et al. ${ }^{12}$ nas enfermarias de 245 hospitais nos Estados Unidos (23,2\%). De acordo com a Sociedade Interamericana de Cardiologia, na América Latina, o baixo investimento na saúde, inadequado acesso ao atendimento e acompanhamento insuficiente nos serviços em nível primário ou terciário são potenciais fatores de risco para o desenvolvimento desta patologia ${ }^{13}$. Dados do registro BREATHE (Brazilian Registry of Acute Heart Failure) ${ }^{14}$ revelaram a má aderência à terapêutica básica para a IC como a principal causa de readmissões hospitalares e de elevada taxa de mortalidade intra-hospitalar, posicionando o Brasil como uma das mais elevadas taxas no mundo ocidental. Para a Sociedade Brasileira de Cardiologia, nosso país apresenta ainda controle inadequado de hipertensão arterial e diabetes, doenças também presentes entre nossos avaliados, e que contribuem diretamente para o desenvolvimento de outras morbidades cardiovasculares ${ }^{15}$.

Um outro aspecto importante observado neste estudo foi o tempo de permanência hospitalar prolongado, pois segundo o Sistema de Informação Hospitalar do Sistema Único de Saúde (SIH-SUS), a média do país é de 5,3 dias ${ }^{16}$. Cabe-se destacar que, um maior tempo de hospitalização tem implicações no custo para o hospital, assim como possíveis 
resultados adversos à saúde do paciente. Dessa forma, verificar os possíveis motivos que levam ao longo tempo de internação é extremamente importante, considerando a qualidade assistencial à saúde, bem como o retrabalho e gasto financeiro das instituições ${ }^{17}$.

Quanto ao desfecho clínico, a exemplo do estudo de Tatsch et al. ${ }^{18}$ realizado em um hospital de ensino do interior do estado do Rio Grande do Sul participante do projeto nutriDia Brasil, nos anos de 2015 e 2016, a maioria dos pacientes receberam alta hospitalar, corroborando com achados da nossa pesquisa (93,3\% e $77,8 \%$, respectivamente vs. $88,2 \%)$.

$\mathrm{O}$ estado nutricional de pacientes internados influencia diretamente no seu tratamento e na sua perspectiva de alta (nutricional e médica), além de estar associado com o risco de complicações durante a permanência ${ }^{19}$. De acordo com - IMC foi possível constatar que os pacientes avaliados foram classificados com sobrepeso, diferente do encontrado no estudo nutriDia Brasil, realizado em Salvador (BA), no qual o IMC médio revelou normalidade $\left(22,83 \mathrm{~kg} / \mathrm{m}^{2}\right)^{20}$. Esta realidade, embora paradoxal, parece traduzir, em nível hospitalar, a situação de transição nutricional vivida em nosso país na atualidade. Verifica-se que, apesar dos índices de desnutrição hospitalar permanecerem altos, com graves consequências para os pacientes, dados recentes têm apontado que mais da metade da população adulta brasileira $(55,7 \%)$ está com excesso de peso ${ }^{21}$.

Por conseguinte, foi observado um percentual expressivo $(54,9 \%)$ de perda de peso não intencional nos três meses precedentes à internação, além de mais de um terço $(34,6 \%)$ terem detalhado em prontuário a presença de risco nutricional ou desnutrição. Essa estimativa de desnutrição é semelhante aos resultados de um programa de melhoria da qualidade publicado recentemente nos Estados Unidos, demonstrando um risco de desnutrição de $30 \%$ em pacientes hospitalares ${ }^{22}$. De acordo com a Academia de Nutrição e Dietética e Sociedade Americana de Nutrição Parenteral e Enteral (ASPEN), a perda de peso involuntária em um curto intervalo de tempo é o parâmetro mais preciso na identificação do risco de desnutrição, por isso, esse fator pré-existente deve ser observado precocemente, a fim de se evitar que esta situação de vulnerabilidade do paciente seja agravada durante a hospitalização ${ }^{23}$.

Sobre o consumo de alimentos a nível domiciliar, foi observado que a maioria dos participantes $(60,8 \%)$ relatou apetite preservado, com consumo normal ou até mais que o normal, resultado superior ao de César et al. ${ }^{9}$, onde mais da metade $(57,3 \%)$ dos pacientes informou ingestão inferior ao habitual. Contudo, com a hospitalização, 59,7\% dos pacientes observados referiram redução do apetite.

Nas enfermarias, a via de dieta predominante do nosso estudo foi a via oral, seguida de nutrição enteral. No nutriDia Brasil de Pelotas, os pesquisadores também encontraram $91,4 \%(n=85)$ dos pacientes utilizando a nutrição via oral e
$8,6 \%(n=8)$, a nutrição enteral ${ }^{9}$. Neste mesmo estudo, menos de um terço dos pacientes referiram ingerir por completo uma das refeições (desjejum, almoço ou jantar) oferecidas no dia da pesquisa, diferindo do nosso estudo, onde pouco mais da metade dos pacientes consumiram a refeição inteira. Contudo, 48, $1 \%$ tiveram inadequação do consumo alimentar (ingestão de $50 \%$ ou menos), estando entre os fatores que influenciaram para tal razão a presença de sintomas gastrointestinais, a insatisfação com as preparações alimentares e as interrupções das refeições ou jejuns para realização de exames e procedimentos cirúrgicos.

Kondrup et al. ${ }^{24}$ demonstraram, em um estudo com pacientes internados, que o consumo de mais de $75 \%$ das necessidades energéticas parece fundamental, uma vez que todos os pacientes que ingeriram abaixo disto apresentaram perda ponderal. Ainda neste contexto, dados provenientes do projeto nutriDia, conduzido em hospitais europeus, encontraram que menos da metade dos pacientes internados consome $100 \%$ da refeição oferecida, e que esses pacientes apresentam maior tempo de hospitalização e risco de óbito, comparado àqueles que ingerem por completo a refeição oferecida ${ }^{25}$. Frente a estes achados e a baixa satisfação dos pacientes em relação à dieta oferecida observada em nosso estudo, reforça-se que, além da prescrição de um adequado aporte calórico-proteico, o monitoramento do consumo real e de aspectos como sabor, cor, aroma, temperatura dos alimentos, horário de distribuição e ambiente aonde se dá a refeição tornam-se parte essencial do cuidado nutricional, de forma a assegurar uma terapia nutricional adequada em relação aos requerimentos nutricionais e que o prescrito esteja sendo efetivamente ingerido.

Um outro aspecto importante observado neste estudo foi que $68,1 \%$ dos pacientes receberam consulta com especialista em nutrição. Porém, 25,9\% dos pacientes tiveram uma intervenção nutricional desenvolvida e 11,3\%, prescrição de terapia nutricional. Os dados do NutritionDay dos Estados Unidos, da mesma forma, também demonstraram que o risco de desnutrição é frequente $(32,7 \%)$ e clinicamente relevante e que a terapia nutricional é subutilizada em pacientes hospitalares, visto que apenas $13,8 \%$ dos avaliados fizeram uso de suplementos orais ou nutrição artificial (enteral ou parenteral), além dos alimentos normais ${ }^{22}$. Esses resultados são preocupantes, pois podem refletir a falta de reconhecimento de pacientes que comem mal e como resultado uma oportunidade perdida de se implementar uma intervenção nutricional precoce e adequada no contexto da rotina hospitalar.

Embora o estudo tenha pontos fortes, convém destacar algumas limitações, como, por exemplo, a avaliação da quantidade de dieta consumida e do histórico de perda de peso corporal, que foram autorreferidos pelos pacientes, o que pode resultar em viés de informação. Os questionários não incluíram dados como profissão, escolaridade e situação conjugal, fatores que podem influenciar no estado nutricional destes. 


\section{CONCLUSÃO}

Tendo por pressuposto que o estado nutricional manifesta por um lado o perfil nutricional da população e, por outro, problemas nutricionais associados à internação, pacientes hospitalizados de um modo geral deveriam ter um acompanhamento dietoterápico estabelecido entre as rotinas hospitalares. Em nosso estudo, a perda ponderal, a inadequação da ingestão alimentar e a presença de desvios nutricionais foram os fatores evidenciados mais relevantes; e esses resultados sugerem que ainda há espaço para implementação de melhorias que contribuam para uma assistência segura e de qualidade ao paciente. Neste contexto, o projeto nutriDia Brasil torna-se uma excelente estratégia para identificação do risco nutricional no âmbito hospitalar, favorecendo a uma intervenção nutricional precoce e melhoria dos resultados alcançados.

\section{REFERÊNCIAS}

1. Correia MITD, Perman MI, Waitzberg DL. Hospital malnutrition in Latin America: a systematic review. Clin Nutr. 2017;36(4):958-67.

2. Waitzberg DL, Caiaffa WT, Correia MI. Hospital malnutrition: the Brazilian national survey (IBRANUTRI): a study of 4000 patients. Nutrition. 2001;17(7-8):573-80.

3. Zheng H, Huang Y, Shi Y, Chen W, Yu J, Wang X. Nutrition status, nutrition support therapy, and food intake are related to prolonged hospital stays in China: results from the Nutrition Day 2015 Survey. Ann Nutr Metab. 2016;69(3-4):215-25.

4. Correia MI, Waitzberg DL. The impact of malnutrition on morbidity, mortality, length of hospital stay and costs evaluated through a multivariate model analysis. Clin Nutr. 2003;22(3):235-9.

5. Araújo Junqueira L, Souza DA. Enteral nutrition therapy for critically ill adult patients: critical review and algorithm creation. Nutr Hosp. 2012;27(4):999-1008.

6. Bottoni A, Hassan DZ, Nacarato A, Garnes AS, Bottoni A. Porque se preocupar com a desnutrição hospitalar?: revisão de literatura. J Health Sci Inst. 2014;32(3):314-7.

7. Kondrup J, Allison SP, Elia M, Vellas B, Plauth M. Educational and Clinical Practice Committee, European Society of Parenteral and Enteral Nutrition (ESPEN). ESPEN guidelines for nutrition screening 2002. Clin Nutr. 2003;22(4):415-21.

8. Nutrition Day Worlwide. Benchmark \& monitor your nutrition care. [cited 2019 Sep 29]. Available from: http://www. nutritionday.org/

9. César JG, Muniz LC, Camargo ACR, Palomino IMV, Garcia $\mathrm{RS}$, Gonzalez MC, et al. Impacto da hospitalização no consumo alimentar de pacientes: resultados do NutriDia Brasil na cidade de Pelotas - RS. Rev Bras Nutr Clín. 2013;28(3):226-30.

10. Brasil. Ministério da Saúde. Secretaria de Vigilância em Saúde. Departamento de Análise de Situação em Saúde. Perfil da saúde no homem. In: Saúde Brasil 2007: uma análise da situação de saúde. Brasília: Ministério da Saúde; 2007. p.509-36.
11. Oliveira MM, Daher DV, Silva JL, Andrade SS. Men's health in question: seeking assistance in primary health care. Cien Saude Colet. 2015;20(1):273-8.

12. Sauer AC, Goates S, Malone A, Mogensen KM, Gewirtz G, Sulz I, et al. Prevalence of malnutrition risk and the impact of nutrition risk on hospital outcomes: results from nutritionDay in the U.S. JPEN J Parenter Enteral Nutr. 2019;43(7):918-26.

13. Bocchi EA, Arias A, Verdejo H, Diez M, Gómez E, Castro P; Interamerican Society of Cardiology. The reality of heart failure in Latin America. J Am Coll Cardiol. 2013;62(11):949-58.

14. Albuquerque DC, Souza Neto JD, Bacal F, Rohde LEP, Bernardez-Pereira S, Berwanger O, et al; Investigadores Estudo BREATHE. I Brazilian Registry of Heart Failure: clinical aspects, care quality and hospitalization outcomes. Arq Bras Cardiol. 2015;104(6):433-42.

15. Rohde LE, Montera MW, Bocchi EA, Clausell N, Albuquerque DC, Rassi S. Diretriz brasileira de insuficiência cardíaca crônica e aguda. Arq Bras Cardiol. 2018;111(3):436-539.

16. Brasil. Ministério da Saúde. Tabnetwin32 3.0: Internações hospitalares do SUS - por local de internação - Brasil. Sistema de informações hospitalares do SUS (SIH/SUS). DATASUS. 2019. [cited 2019 Nov 17]. Available from: http:// http://tabnet. datasus.gov.br/cgi/tabcgi.exe?sih/cnv/sxuf.def

17. Rufino GP, Gurgel MG, Pontes TC, Freire E. Avaliação de fatores determinantes do tempo de internação em clínica médica. Rev Bras Clin Med. 2012;10(4):291-7.

18. Tatsch CG, Fischer MQ, Dalmonte KS, BaelzK, Poll FA, Antunes CT. NutriDia Brasil: a atuação de um hospital de ensino nos anos de 2015 e 2016. Rev Saúde (Sta. Maria). 2018;44(2):1-8.

19. Waitzberg DL, Aguilar-Nascimento JE, Dias MCG, Pinho N, Moura R, Correia MITD. Hospital and homecare malnutrition and nutritional therapy in Brazil. Strategies for alleviating it: a position paper. Nutr Hosp. 2017;34(4):969-75.

20. Sampaio EJ, Meirelles ARN, Gusmão MHL, Cruz IS, Luedy A. Participação do Hospital Universitário no NUTRIDIA Brasil 2012: estratégia para a assistência segura ao paciente. Rev Acred. 2015;5(9):21-9.

21. Brasil. VigitelBrasil2018: vigilância de fatores deriscoe proteção para doenças crônicas por inquérito telefônico. Brasília: Ministério da Saúde; 2019.

22. Sherry CL, Sauer AC, Thrush KE. Assessment of the nutrition care process in US hospitals using a web-based tool demonstrates the need for quality improvement in malnutrition diagnosis and discharge care. Curr Dev Nutr. 2017;1(11):e001297.

23. White JV, Guenter P, Jensen G, Malone A, Schofield M; Academy Malnutrition Work Group; A.S.P.E.N. Malnutrition Task Force; A.S.P.E.N. Board of Directors. Consensus statement: Academy of Nutrition and Dietetics and American Society for Parenteral and Enteral Nutrition: characteristics recommended for the identification and documentation of adult malnutrition (undernutrition). JPEN J Parenter Enteral Nutr. 2012;36(3):275-83.

24. Kondrup J, Johansen N, Plum LM, Bak L, Larsen IH, Martinsen $\mathrm{A}$, et al. Incidence of nutritional risk and causes of inadequate nutritional care in hospitals. Clin Nutr. 2002;21(6):461-8.

25. Hiesmayr M, Schindler K, Pernicka E, Schuh C, Schoeniger-Hekele A, Bauer P, et al; NutritionDay Audit Team. Decreased food intake is a risk factor for mortality in hospitalised patients: the NutritionDay survey 2006. Clin Nutr. 2009;28(5):484-91. 